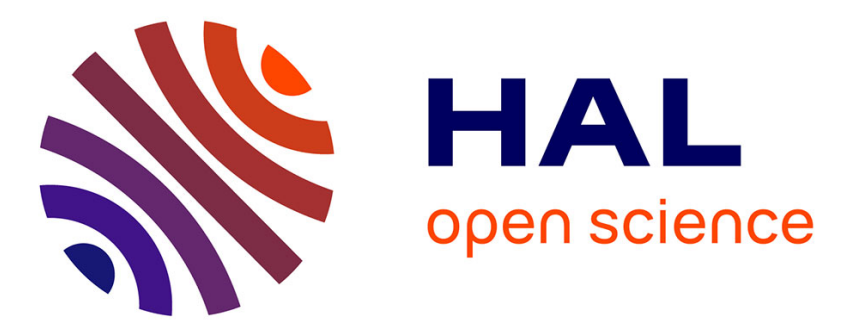

\title{
Réévaluation du sexe et de l'âge au décès du sujet azilien Le Peyrat 5, Saint-Rabier (Dordogne, France)
}

Mathilde Samsel, Christopher J. Knüsel, Sébastien Villotte

\section{To cite this version:}

Mathilde Samsel, Christopher J. Knüsel, Sébastien Villotte. Réévaluation du sexe et de l'âge au décès du sujet azilien Le Peyrat 5, Saint-Rabier (Dordogne, France). Bulletins et Mémoires de la Société d'anthropologie de Paris, 2016, 28, pp.213-220. 10.1007/s13219-016-0162-9 . hal-03146937

\section{HAL Id: hal-03146937 \\ https://hal.science/hal-03146937}

Submitted on 26 Feb 2021

HAL is a multi-disciplinary open access archive for the deposit and dissemination of scientific research documents, whether they are published or not. The documents may come from teaching and research institutions in France or abroad, or from public or private research centers.
L'archive ouverte pluridisciplinaire HAL, est destinée au dépôt et à la diffusion de documents scientifiques de niveau recherche, publiés ou non, émanant des établissements d'enseignement et de recherche français ou étrangers, des laboratoires publics ou privés. 


\title{
Réévaluation du sexe et de l'âge au décès du sujet azilien Le Peyrat 5, Saint-Rabier (Dordogne, France)
}

\section{Reassessment of the Sex and Age-at-Death of the Azilian Skeleton from Le Peyrat 5, Saint-Rabier (Dordogne, France)}

\author{
M. Samsel · C.J. Knüsel · S. Villotte \\ Reçu le 10 mai 2016; accepté le 30 juin 2016 \\ C) Société d'Anthropologie de Paris et Lavoisier SAS 2016
}

\begin{abstract}
Résumé Cette note propose une réévaluation de la diagnose sexuelle et de la détermination de l'âge au décès de l'individu azilien Le Peyrat 5 (LP5) [Dordogne, France] daté directement à $11430 \pm 150$ BP (GifA-99117; 13566-13017 cal. $\mathrm{BP})$ à partir de méthodes fiables appliquées à l'os coxal. Cette étude confirme que le squelette LP5 est celui d'un homme adulte vraisemblablement décédé entre 30 et 49 ans. La détermination fiable du sexe de vestiges du Paléolithique supérieur est notamment importante pour la construction d'un corpus de référence visant à mieux appréhender le dimorphisme sexuel extra-pelvien des individus de cette période.
\end{abstract}

Mots clés Diagnose sexuelle - Os coxal · Épipaléolithique · Âge au décès · Méthode

\begin{abstract}
This note proposes reassessment of the sex and age-at-death of the Azilian individual Le Peyrat 5 (Dordogne, France) directly dated to $11,430 \pm 150$ BP (GifA99117; 13,566-13,017 cal. BP) using reliable methods applied to the os coxae. This study confirms that the Le Peyrat 5 skeleton is that of an adult male who was between 30 and 49 years of age at death. Reliable sex determination is especially important for the construction of a reference corpus of Upper Paleolithic individuals which permits a better appreciation of extra-pelvic sexual dimorphism of individuals dating from these periods.
\end{abstract}

Keywords Sex determination - Os coxae - Epipaleolithic Age-at-death $\cdot$ Method

\footnotetext{
M. Samsel $(\bowtie) \cdot$ C.J. Knüsel

Université de Bordeaux, PACEA,

UMR 5199, F-33615 Pessac, France

e-mail : mathilde.samsel@u-bordeaux.fr

S. Villotte

CNRS, Université de Bordeaux, PACEA,

UMR 5199, F-33615 Pessac, France
}

\section{Introduction}

Le gisement en grotte du Peyrat est localisé sur le territoire de la commune de Saint-Rabier en Dordogne, France. Le matériel archéologique attribué au Magdalénien supérieur, à l'Azilien et au Sauveterrien est issu des fouilles menées par le Dr Cheynier de 1958 à 1967 [1,2]. Ces travaux sont renseignés par quelques notes ponctuelles et un carnet de fouilles à partir desquels Cleyet-Merle [2] a proposé un bilan et des interprétations. Le Catalogue of Fossil Hominids [3] recense les restes humains du Peyrat sous les entrées « Le Peyrat » et « Saint-Rabier », rendant leur inventaire confus. Un nombre de huit individus est retenu pour l'ensemble des vestiges anthropologiques retrouvés sur le site, toutes provenances stratigraphiques confondues [3]. Des vestiges fragmentaires crâniens et dentaires sont attribués au Magdalénien supérieur (LP1 à LP4 ${ }^{1}$ ). Le niveau sauveterrien a livré une prémolaire isolée dénommée « Saint-Rabier 1 » [3-6]. Deux squelettes adultes (LP5 et LP6) ainsi qu'un fragment de mandibule (LP7) ont été découverts dans le niveau ANV correspondant à une couche stratigraphique de la grotte supérieure (extrémité ouest de la tranchée $\mathrm{N}$ ) « sous la voûte » [2]. Ce niveau fortement remanié est attribué à l'Azilien dans les notes du Dr Cheynier, sans que rien de typique dans l'assemblage lithique ne permette de confirmer cette attribution selon Cleyet-Merle [2]. Seuls les deux squelettes LP5 et LP6 ont directement fait l'objet d'une datation radiocarbone par spectrométrie de masse par accélérateur [7]. LP6, correspondant à un individu moyennement représenté, est attribué au début de la période médiévale avec une date de $520 \pm 80$ AD (GifA-99118; [7]). Il s'agit vraisemblablement d'un dépôt intrusif dans un niveau remanié contenant à la fois des silex paléolithiques et des poteries [2]. LP5 correspond au squelette subcomplet et bien conservé d'un individu adulte. Il a été daté à $11430 \pm 150$ BP (GifA-99117 ;

\footnotetext{
$\overline{1}$ « LP » pour $«$ Le Peyrat ».
} 
13566-13017 cal. $\mathrm{BP}^{2} ; 13 \mathrm{C}:-18,2: 15 \mathrm{~N}: 12,1$ [7]). Cette date tend à confirmer son attribution à l'Azilien (entre 14000 et 12500 cal. BP environ).

LP5 appartient aujourd'hui aux collections du musée Labenche de la Ville de Brive (Corrèze, France) et y est présenté de manière permanente au sein de la salle Préhistoire. Il a fait l'objet en 1968 d'une étude descriptive par Patte, qui a estimé l'âge au décès de ce sujet à partir de l'observation du degré de synostose des sutures crâniennes : «la sagittale est fermée à une certaine distance au-delà du bregma ; la lambdoïde l'est en partie. Étant donné les variations individuelles, il est difficile de conclure avec précision, on peut admettre une quarantaine d'années sans garantir cette estimation » [6]. Le sexe de LP5 a également été déterminé selon la morphologie de ses structures crâniennes, dont «les reliefs accusés ne laissent pas de doute sur l'attribution à un homme » [6] (Fig. 1). Patte ne fait mention d'aucune méthode utilisée, ses descriptions restant sommaires, et il semble que seule son expérience permette de conclure sur l'estimation de l'âge au décès et du sexe de LP5.

L'estimation de l'âge au décès des adultes à partir du squelette reste problématique, du fait d'une variabilité individuelle importante face aux processus de sénescence $[10,11]$. Toutefois, nous savons à l'heure actuelle que l'observation du degré de synostose des sutures crâniennes pour proposer un âge au décès est peu performante [10] et que la surface sacro-pelvienne iliaque est l'un des meilleurs indicateurs osseux $[10,11]$.

Les caractères morphologiques crâniens pris isolément restent des indicateurs médiocres dans la diagnose sexuelle. De plus, le dimorphisme sexuel extra-pelvien des populations actuelles ne correspondant pas à celui existant pour des populations préhistoriques, ces dernières présentant une plus forte robustesse, l'utilisation de ce type de méthodes peut mener à des diagnoses sexuelles erronées et à un fort taux d'erreur [12-16]. À l'inverse, les variations de forme liées aux contraintes fonctionnelles qu'entraînent la bipédie et la reproduction induisent un dimorphisme sexuel pelvien commun à toutes les populations d'Homo sapiens. Ainsi, les os coxaux constituent le meilleur indicateur osseux pour déterminer le sexe d'un squelette d'homme moderne adulte [17].

L'absence de diagnostic sexuel et d'estimation de l'âge fiables est problématique dans la perspective d'analyses paléobiologiques individuelles et populationnelles ainsi que dans l'interprétation des gestes funéraires. Il semblait donc nécessaire de proposer la réévaluation de l'estimation de l'âge au décès et du sexe du squelette LP5 sur des bases méthodologiques récentes les plus fiables possible.

\footnotetext{
${ }^{2}$ Date calibrée selon la courbe IntCal13 dans le logiciel Calib Rev7.1.0 $[8,9]$.
}

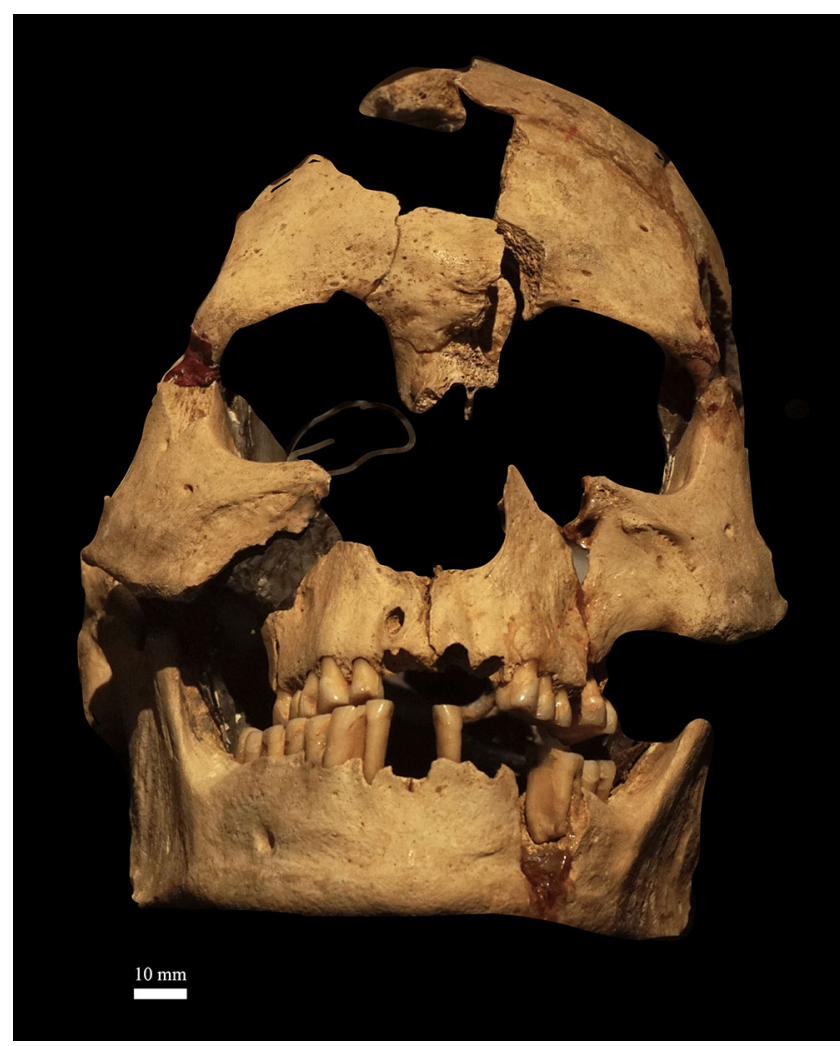

Fig. 1 Crâne et mandibule du sujet LP5, norma facialis (collection du musée Labenche - Ville de Brive ; photographie : M. Samsel) / The cranium and mandible of LP5, norma facialis (Collection of musée Labenche - Ville de Brive; photograph: M. Samsel)

\section{Matériel et méthodes}

Le squelette LP5 est globalement bien représenté et conservé (Fig. 2). Les os coxaux sont particulièrement bien conservés (Fig. 3), rendant possible l'application des méthodes d'estimation de l'âge et de détermination du sexe sur le coxal.

L'estimation de l'âge au décès de LP5 repose sur l'observation du degré de fusion des points d'ossification secondaires, en particulier les plus tardifs (crête iliaque et extrémité sternale de la clavicule [18]), ainsi que sur l'observation des caractéristiques morphologiques de la surface sacropelvienne iliaque cotées suivant la méthode proposée par Schmitt [19]. Néanmoins, l'aspect des caractéristiques retenues dans cette méthode est polyfactoriel (génétique, culture, environnement). Fiable pour des populations européennes [19], l'application de la méthode de Schmitt à des individus préhistoriques génétiquement et culturellement différents de l'échantillon de référence utilisé lors de l'élaboration de la méthode pose question. Bien que cela soit peu satisfaisant, il est alors nécessaire de faire l'hypothèse que "le mode de sénescence des marqueurs ostéologiques est resté inchangé depuis l'apparition de l'homme moderne » [19], hypothèse déjà formulée lors de l'application de cette 


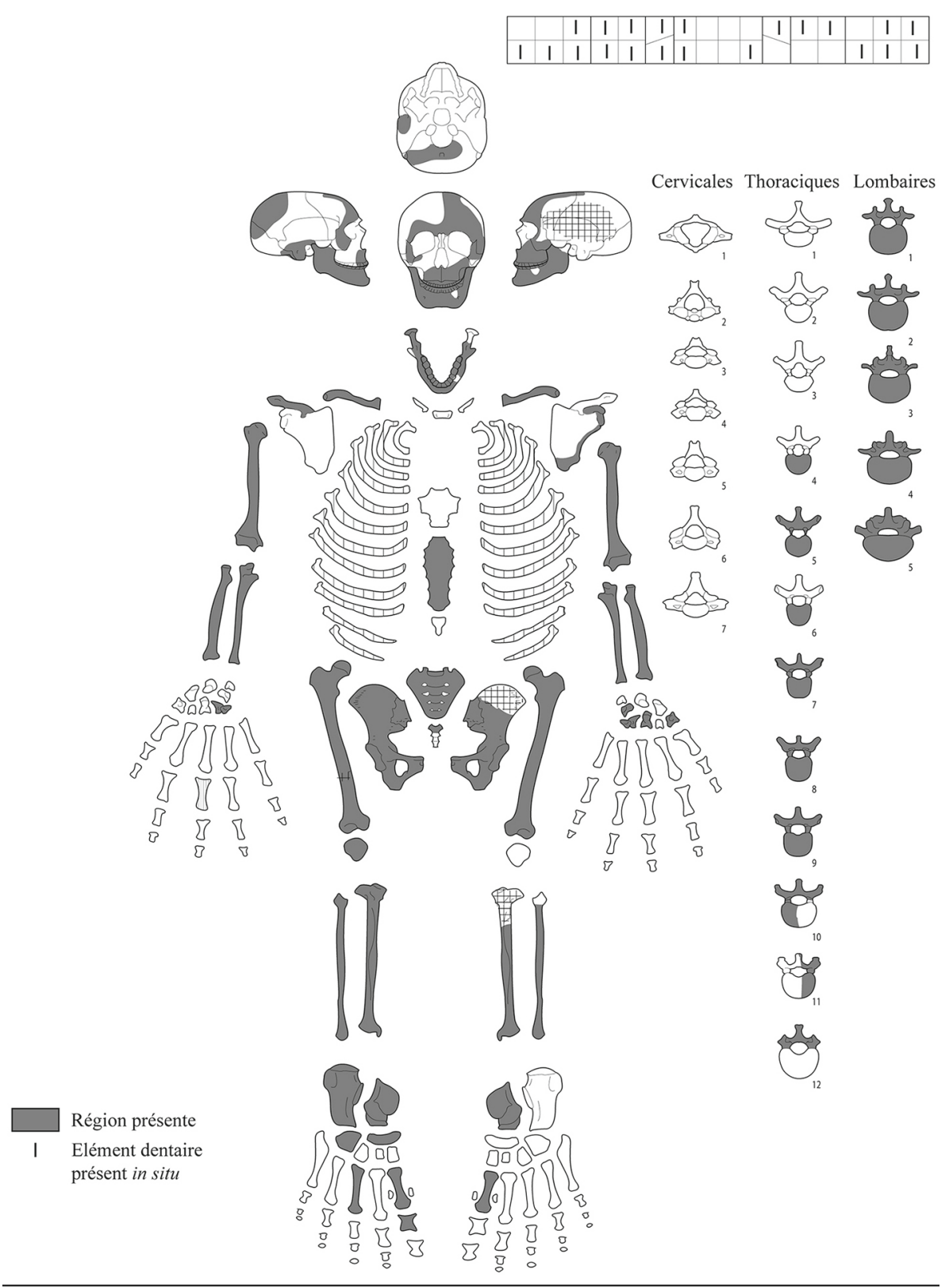

Fig. 2 Diagramme montrant la représentation et la conservation du squelette LP5. La mandibule a été fixée avec le crâne qui est partiellement représenté. L'ensemble des os longs des membres ainsi que les clavicules sont conservés / Diagram showing preservation of the skeleton LP5 (bones in grey are present). The partially preserved cranium and the mandible are fixed together. All long bones of limbs and clavicles are preserved

méthode à des vestiges du Paléolithique supérieur [20,21]. La présence ou non d'atteintes dégénératives du squelette ainsi que le degré d'usure dentaire [22] ont également été considérés comme des indicateurs.

La détermination du sexe de LP5 repose sur la méthode morphoscopique [23] et la diagnose sexuelle probabiliste (DSP) [24] appliquées sur l'os coxal. Ces deux méthodes atteignent toutes les deux une fiabilité minimum de $95 \%$ de détermination correcte. Elles ont déjà été appliquées à des vestiges du Paléolithique supérieur et du début de l'Ho- locène [16,20,21,25] (comm. pers. M. Samsel) (Tableau 1). Pour certains de ces vestiges, des analyses génomiques ont confirmé cette diagnose [26,27].

\section{Résultats}

\section{Estimation de l'âge au décès}

La totalité des épiphyses préservées des os longs du squelette LP5 sont fusionnées aux diaphyses. La synchondrose 


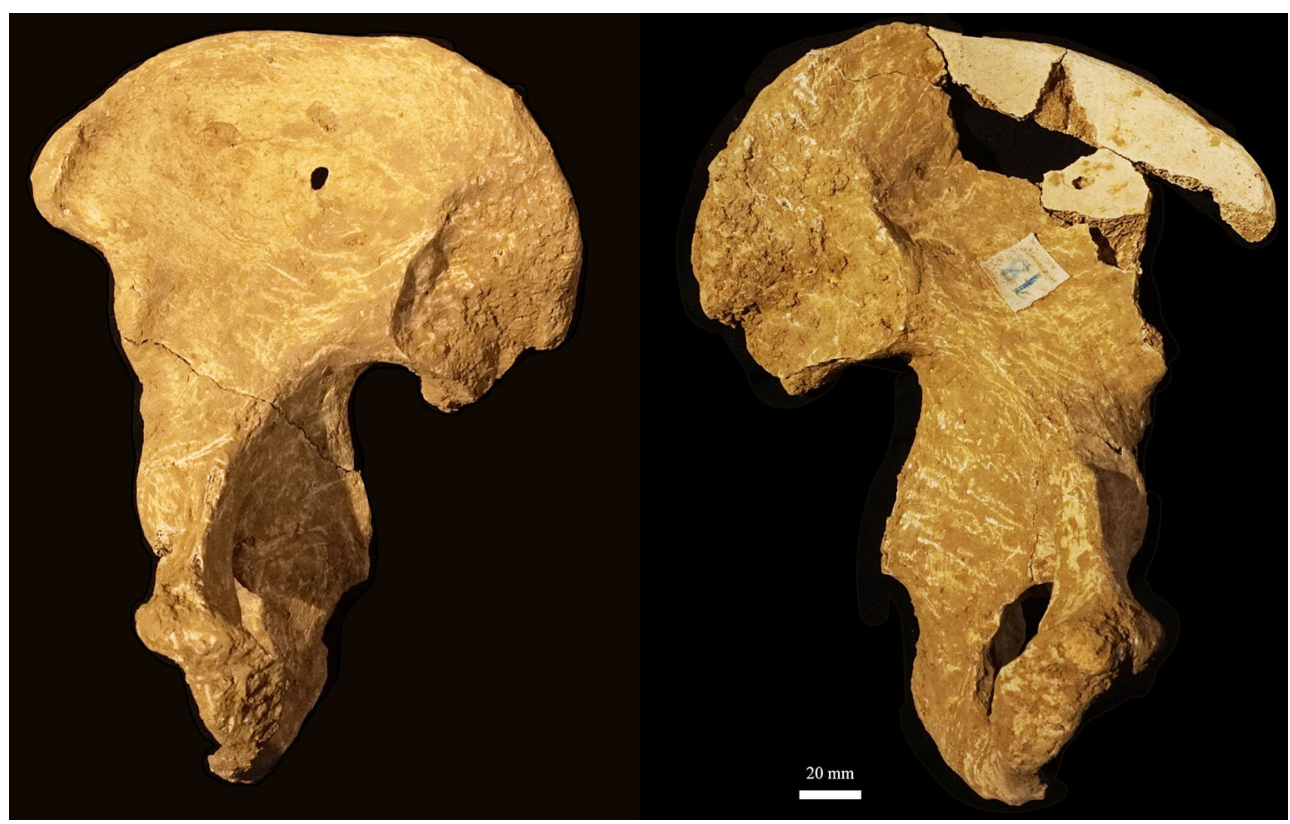

Fig. 3 Os coxal droit (à gauche de l'image) et os coxal gauche (à droite de l'image) du sujet LP5, vue médiale (collection du musée Labenche - Ville de Brive ; photographie : M. Samsel) / Right os coxae (on the left) and left os coxae (on the right) of LP5, medial view (Collection of musée Labenche - Ville de Brive; photograph: M. Samsel)

Tableau 1 Corpus d'individus bien conservés datés de la fin du Paléolithique supérieur pour lesquels une diagnose sexuelle primaire est disponible à partir des méthodes développées sur l'os coxal [23,24] et/ou par analyse du chromosome Y / Corpus of wellpreserved individuals dated from the end of the Upper Palaeolithic with a sex assessment using methods applied to the os coxae $[23,24]$ and / or by Y-chromosome analysis.

\begin{tabular}{|c|c|c|}
\hline Individu & Culture & Sexe \\
\hline El Miron & Magdalénien & $\mathrm{F}^{\mathrm{b}}[27]$ \\
\hline Lafaye & Magdalénien & $\mathrm{F}[21]$ \\
\hline Saint-Germain-la-Rivière & Magdalénien & $\mathrm{F}[25]$ \\
\hline Arene Candide 3 & Épigravettien & M [21] \\
\hline Arene Candide 4 & Épigravettien & M [21] \\
\hline Arene Candide 5 & Épigravettien & $\mathrm{M}^{\mathrm{b}}[28]$ \\
\hline Arene Candide 12 & Épigravettien & $\mathrm{M}[21]$ \\
\hline Le Bichon & Azilien & $\mathrm{M}^{\mathrm{a}}[21,26]$ \\
\hline Continenza 7 & Épigravettien & M [21] \\
\hline Grotte des Enfants 3 & Épigravettien & $\mathrm{F}[21]$ \\
\hline Romanelli 1 & Épigravettien & $\mathrm{M}[21]$ \\
\hline Romito 3 & Épigravettien & M [21] \\
\hline Romito 6 & Épigravettien & M [21] \\
\hline Romito 7 & Épigravettien & M [21] \\
\hline San Teodoro 1 & Épigravettien & $\mathrm{F}[21]$ \\
\hline San Teodoro 4 & Épigravettien & $\mathrm{F}[21]$ \\
\hline Tagliente 2 & Épigravettien & M [21] \\
\hline Vado all'Arancio & Épigravettien & M [21] \\
\hline Vasilyevka III 12 & Épipaléolithique & $\mathrm{F}[21]$ \\
\hline Vasilyevka III 36 & Épipaléolithique & $\mathrm{M}[21]$ \\
\hline Villabruna 1 & Épigravettien & $\mathrm{M}^{\mathrm{a}}[21,27]$ \\
\hline
\end{tabular}


sphéno-occipitale ainsi que le listel des vertèbres conservées sont également totalement soudés. La crête iliaque des deux coxaux et l'extrémité sternale des deux clavicules sont totalement fusionnées. Ces éléments permettent de conclure à la présence d'un individu adulte.

La méthode de cotation des caractéristiques morphologiques de la surface sacro-pelvienne iliaque permet d'estimer un âge au décès pour ce sujet entre 20 et 49 ans à $98 \%$ de probabilité.

La présence de changements dégénératifs de type arthrose au niveau de la cavité glénoïde de la scapula gauche, des métatarsiens, des vertèbres thoraciques médianes ainsi que des vertèbres lombaires plaide pour un âge adulte assez avancé. Les dents conservées montrent un degré d'usure dentaire modéré à fort. L'ensemble de ces éléments semble justifier de conserver la partie haute de l'intervalle chronologique obtenu à partir de la cotation des caractéristiques morphologiques de la surface sacro-pelvienne, avec un décès vraisemblablement survenu entre 30 et 49 ans.

\section{Détermination du sexe}

Que l'on considère l'os coxal gauche ou le droit, la méthode morphoscopique détermine LP5 comme un individu masculin (Tableau 2). Les probabilités pour LP5 d'être un individu masculin sont d'après la DSP de $99 \%$ pour l'os coxal gauche et de $100 \%$ pour l'os coxal droit (Tableau 3).

\section{Discussion et conclusions}

Le réexamen des os coxaux du squelette LP5 confirme, au moyen de méthodes dont la fiabilité est connue, l'attribution de cet individu à un homme adulte.

En paléoanthropologie, l'estimation de l'âge au décès reste problématique. Il n'existe pas de méthode qui ne soit pas « population-dépendante ». Ainsi, la méthode de Schmitt ne reste fiable que pour des populations européennes et modernes $[11,19]$. Toutefois, en tenant également compte d'indicateurs tels le degré de fusion de certains points d'ossification secondaires du squelette, les atteintes dégénératives ou encore l'usure dentaire, il semble vraisemblable de proposer pour LP5 un décès survenu entre 30 et 49 ans.

Les deux méthodes fondées sur l'os coxal permettent de conclure de façon fiable à un sexe masculin pour l'individu azilien LP5. Ces résultats sont en accord avec l'aspect robuste et les principales dimensions de LP5 (Tableaux 4, 5). La diagnose sexuelle de LP5 à partir des éléments pelviens permet de confirmer la détermination de Patte [6] effectuée à partir de sa morphologie crânienne. Les os coxaux des individus datant du Paléolithique supérieur sont souvent fragmentaires ou absents, ne permettant pas une diagnose sexuelle à partir des éléments pelviens. Ainsi, une part importante des squelettes bien conservés pour ces périodes restent de sexe indéterminé de façon primaire. Outre les analyses génétiques portant sur le chromosome $\mathrm{Y}$, seule une diagnose sexuelle secondaire, fondée sur un dimorphisme

Tableau 2 Résultats de la diagnose sexuelle de LP5 selon la méthode morphoscopique [23] / Results of the sex diagnosis of LP5 with the method for visual determination using the human hip bone [23].

\begin{tabular}{|c|c|c|c|c|c|}
\hline \multirow[t]{2}{*}{ Caractère } & \multirow[t]{2}{*}{ Sous-caractère } & \multicolumn{2}{|l|}{ Coxal gauche } & \multicolumn{2}{|l|}{ Coxal droit } \\
\hline & & $\begin{array}{l}\text { Résultat sous- } \\
\text { caractère }\end{array}$ & $\begin{array}{l}\text { Résultat } \\
\text { caractère }\end{array}$ & $\begin{array}{l}\text { Résultat sous- } \\
\text { caractère }\end{array}$ & $\begin{array}{l}\text { Résultat } \\
\text { caractère }\end{array}$ \\
\hline Région & Absence ou présence de dépression & $\mathrm{m}$ & $\mathbf{M}$ & $\mathrm{m}$ & $\mathbf{M}$ \\
\hline \multirow[t]{2}{*}{ Préauriculaire } & Caractéristique de la dépression & $\mathrm{i}$ & & $\mathrm{m}$ & \\
\hline & Tubercule de Buisson & $\mathrm{m}$ & & $\mathrm{m}$ & \\
\hline \multirow{3}{*}{$\begin{array}{l}\text { Grande incisure } \\
\text { ischiatique }\end{array}$} & Longueur relative segments $\mathrm{AC}$ et $\mathrm{BC}$ & $\mathrm{m}$ & $\mathbf{M}$ & $\mathrm{m}$ & $\mathbf{M}$ \\
\hline & Symétrie & $\mathrm{m}$ & & $\mathrm{m}$ & \\
\hline & $\begin{array}{l}\text { Position de la perpendiculaire passant } \\
\text { par A }\end{array}$ & $\mathrm{m}$ & & $\mathrm{m}$ & \\
\hline Arc composé & Incisure et surface auriculaire & $\mathrm{m}$ & $\mathbf{M}$ & $\mathrm{m}$ & $\mathbf{M}$ \\
\hline \multirow[t]{3}{*}{ Bord inférieur } & Incurvation ou non & $\mathrm{m}$ & $\mathbf{M}$ & $\mathrm{m}$ & $\mathbf{M}$ \\
\hline & Crista phallica & $\mathrm{i}$ & & $\mathrm{i}$ & \\
\hline & Branche ischiopubienne & $\mathrm{m}$ & & $\mathrm{m}$ & \\
\hline \multirow{2}{*}{\multicolumn{2}{|c|}{$\begin{array}{l}\text { Longueur relation ischium-pubis } \\
\text { Attribution sexe }\end{array}$}} & $\mathrm{i}$ & I & $\mathrm{i}$ & $\mathbf{I}$ \\
\hline & & Masculin & & Masculin & \\
\hline
\end{tabular}




\begin{tabular}{|c|c|c|c|c|}
\hline Variable & M\#\# & Définition & Côté gauche & Côté droit \\
\hline PUM & M14 & Longueur du pubis & 68,7 & 65,8 \\
\hline SPU & & Largeur pubo-acétabulaire & 29,0 & 30,4 \\
\hline DCOX & M1 & Hauteur maximale du coxal & 216,0 & 218,0 \\
\hline IIMT & M15.1 & Hauteur de la grande incisure ischiatique & 42,7 & 37,8 \\
\hline ISMM & & Longueur de l'ischium & 116,1 & 114,9 \\
\hline SCOX & M12 & Largeur de l'os coxal & 166,0 & 169,0 \\
\hline SS & & Distance spino-sciatique & / & 74,2 \\
\hline SA & & Distance spino-auriculaire & / & 75,4 \\
\hline SIS & M14.1 & Largeur cotylo-sciatique & 39,8 & 41,1 \\
\hline VEAC & M22 & Diamètre vertical de l'acétabulum & 54,5 & 56,8 \\
\hline $\mathrm{P}(\mathrm{F})$ & & & 0,0001 & 0,0000 \\
\hline $\mathrm{P}(\mathrm{M})$ & & & 0,9999 & 1,0000 \\
\hline SEXE & & & Mâle & Mâle \\
\hline \multicolumn{5}{|c|}{ 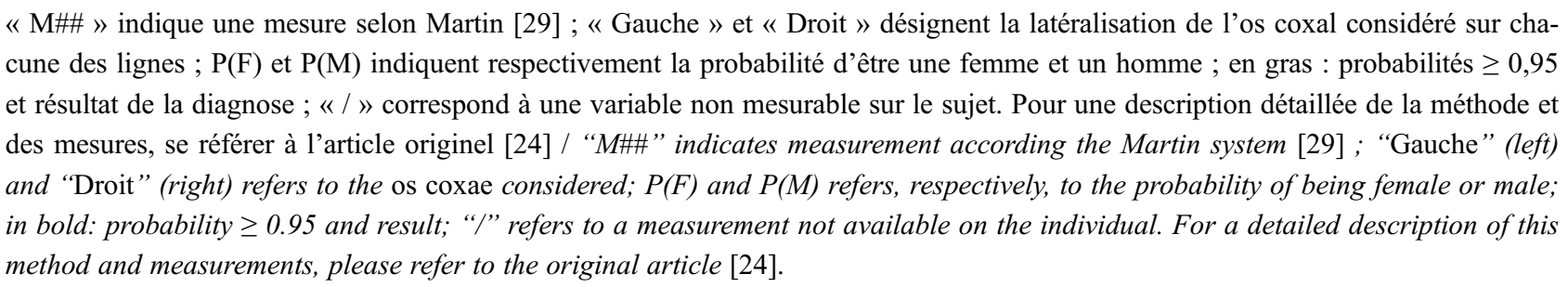 } \\
\hline
\end{tabular}

Tableau 4 Principales mesures crâniennes et mandibulaires de LP5 / Main measurements of the cranium and the skull of LP5.

\begin{tabular}{|c|c|c|c|}
\hline M\#\# & Définition & Mesure & \\
\hline M1 & Longueur maximale crânienne & 192,0 & \\
\hline M8 & Largeur maximale du crâne & 135,0 & \\
\hline M9 & Largeur frontale minimale & 95,0 & \\
\hline M20 & Hauteur porion-bregma & 136,0 & \\
\hline M48 & Hauteur nasale supérieure & 67,0 & \\
\hline M51 & Largeur de l'orbite & $\mathrm{G}: /$ & $\mathrm{D}: 39,0$ \\
\hline M52 & Hauteur de l'orbite & $\mathrm{G}: 31,0$ & $D: 29,6$ \\
\hline M55 & Hauteur de la cavité nasale & 49,0 & \\
\hline M66 & Largeur bi-goniaque & 114,1 & \\
\hline M68 (1) & Longueur de la mandibule avec projection & 107,0 & \\
\hline M68 & Longueur de la mandibule sans projection & 103,0 & \\
\hline M70 & Hauteur de la branche montante en position & $\mathrm{G}: /$ & $\mathrm{D}: 59,0$ \\
\hline M71a & Largeur minimale de la branche montante & $\mathrm{G}: 36,7$ & $\mathrm{D}: 38,3$ \\
\hline \multicolumn{4}{|c|}{ 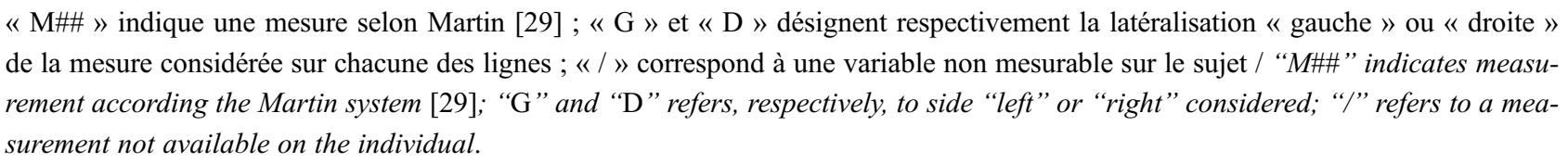 } \\
\hline
\end{tabular}

sexuel de taille et de conformation des éléments osseux infra-crâniens [30], est alors possible. Ce type de démarche nécessite d'avoir un corpus de référence pour lequel un sexe est déterminé à partir de méthodes fiables se focalisant sur l'os coxal, ce corpus devant être spécifique à un groupe chronologiquement et géographiquement homogène $[15,30]$. La constitution d'un tel corpus pour la fin du Pléistocène est donc essentielle. Il permettra d'envisager de nouvelles pistes 
Tableau 5 Principales mesures infra-crâniennes de LP5 / Main infra-cranial measurements of LP5.

\begin{tabular}{|c|c|c|c|}
\hline M\#\# & Définition & Gauche & Droit \\
\hline M1 & Clavicule : longueur maximale de la clavicule & 149,0 & 138,0 \\
\hline M6 & Clavicule : périmètre au milieu & 41,0 & 40,0 \\
\hline M1 & Humérus : longueur maximale & 318,0 & 312,0 \\
\hline M3 & Humérus : largeur de l'extrémité proximale & 51,7 & 51,3 \\
\hline M4 & Humérus : largeur de l’extrémité distale & 62,3 & 60,9 \\
\hline M5 & Humérus : diamètre maximal au milieu & 27,2 & 23,5 \\
\hline M6 & Humérus : diamètre minimal au milieu & 19,9 & 17,3 \\
\hline M7 & Humérus : périmètre minimal de la diaphyse & 73,0 & 64,0 \\
\hline / & Humérus : indice de robustesse $(\mathrm{M} 7 / \mathrm{M} 1 * 100)$ & 23,0 & 20,5 \\
\hline M1 & Radius : longueur maximale & 248,0 & 250,0 \\
\hline M3 & Radius : périmètre minimal & 41,0 & 40,0 \\
\hline M4 & Radius : diamètre transversal maximal de la diaphyse & 17,4 & 17,2 \\
\hline M5 & Radius : diamètre antéro-postérieur au niveau de M4 & 11,6 & 11,8 \\
\hline M1 & Ulna : longueur maximale & 267,0 & 268,0 \\
\hline M3 & Ulna : périmètre minimal de la diaphyse & 37,0 & 36,0 \\
\hline M11 & Ulna : diamètre antéro-postérieur au niveau de M12 & 15,3 & 17,0 \\
\hline M12 & Ulna : diamètre transversal maximal de la diaphyse & 16,8 & 17,3 \\
\hline M1 & Fémur : longueur maximale & 436,0 & 440,0 \\
\hline M2 & Fémur : longueur anatomique & 434,0 & 439,0 \\
\hline M6 & Fémur : diamètre antéro-postérieur au milieu & 35,5 & 35,9 \\
\hline M7 & Fémur : diamètre transversal au milieu & 27,8 & 28,9 \\
\hline M8 & Fémur : périmètre au milieu & 101,0 & 103,0 \\
\hline M9 & Fémur : diamètre transversal sous-trochantérien & 34,1 & 35,1 \\
\hline M10 & Fémur : diamètre antéro-postérieur sous-trochantérien & 29,7 & 29,6 \\
\hline M18 & Fémur : diamètre vertical de la tête articulaire & 46,6 & 47,3 \\
\hline M19 & Fémur : diamètre antéro-postérieur de la tête articulaire & 46,7 & 46,4 \\
\hline M21 & Fémur : largeur de l'extrémité distale & 83,0 & 84,0 \\
\hline / & Fémur : indice de robustesse $(\mathrm{M} 8 / \mathrm{M} 1 * 100)$ & 23,2 & 23,4 \\
\hline M1a & Tibia : longueur maximale & 376,0 & 375,0 \\
\hline M3 & Tibia : largeur de l'extrémité proximale & 1 & 81,0 \\
\hline M6 & Tibia : largeur de l'extrémité distale & 52,2 & 53,0 \\
\hline M8a & Tibia : diamètre antéro-postérieur au niveau du foramen nourricier & 40,5 & 40,0 \\
\hline M9a & Tibia : diamètre transversal au niveau du foramen nourricier & / & 21,9 \\
\hline M10a & Tibia : périmètre au niveau du foramen nourricier & / & 102,0 \\
\hline $\mathrm{M} 10 \mathrm{~b}$ & Tibia : périmètre minimal de la diaphyse & 83,0 & 82,0 \\
\hline
\end{tabular}

pour l'appréhension du dimorphisme sexuel extra-pelvien des populations préhistoriques et de proposer de nouvelles approches d'estimation du sexe d'individus dont les os coxaux sont absents ou trop fragmentaires.

Remerciements Nous adressons nos remerciements au musée Labenche de la Ville de Brive et à Laudine Michelin sa responsable scientifique et technique pour nous avoir permis l'accès et l'étude du sujet Le Peyrat 5. Cette étude a été réalisée dans le cadre d'un contrat doctoral à l'univer- sité de Bordeaux (MS), avec le soutien financier de l'UMR 5199 PACEA, de l'université de Bordeaux (Appel à projets " Recherche » 2014, resp. S. Villotte), du Partenariat Hubert Curien (Programme Tournesol 34228WD, resp. français : S. Villotte, resp. belge : C. Polet) et de l'Agence nationale de la recherche (ANR-15-CE33-0004, resp. : S. Villotte).

Liens d'intérêts : les auteurs déclarent ne pas avoir de lien d'intérêt. 


\section{Références}

1. Cheynier A (1964) Les fouilles du Peyrat, campagne de 1964. Bull Soc Prehist Fr 61:169-70

2. Cleyet-Merle JJ (1988) Le gisement magdalénien du Peyrat à Saint-Rabier (Dordogne) d'après les fouilles Cheynier, 19581967. Bull Soc Prehist Fr 85:332-51

3. Petit-Maire N, Ferembach D, Bouvier JM, et al (1971) France. In: Oakley KP, Campbell BG, Molleson TI (eds) Catalogue of Fossil Hominids, Part II: Europe. Trustees of the British Museum (National History), London, pp 71-187

4. Meiklejohn C, Bosset G, Valentin F (2010) Radiocarbon dating of Mesolithic human remains in France. Mesolithic Miscellany 21:10-56

5. Patte E (1963) Restes d'un enfant et dents du Magdalénien et du Mésolithique de Saint-Rabier (fouilles Cheynier). Anthropologie 67:513-24

6. Patte E (1968) L'homme et la femme de l'Azilien de Saint-Rabier (fouilles Cheynier). Mémoires du Muséum national d'histoire naturelle série C, Sciences de la terre Tome XIX, pp 1-56

7. Drucker D (2001) Validation méthodologique de l'analyse isotopique d'ossements fossiles et apports aux reconstitutions paléoécologiques du Paléolithique supérieur du Sud-Ouest de la France. Thèse de doctorat, université de Paris-VI, Paris, $222 \mathrm{p}$

8. Stuiver M, Reimer PJ, Reimer RW (2005) CALIB 5.0. [program and documentation]. http://calib.qub.ac.uk/calib/

9. Reimer PJ, Bard E, Bayliss A, et al (2013) IntCal13 and Marine13 Radiocarbon AGE Calibration Curves 0-50,000 Years cal BP. Radiocarbon 55:1869-87

10. Schmitt A (2001) Variabilité de la sénescence du squelette humain. Réflexions sur les indicateurs de l'âge au décès : à la recherche d'un outil performant. Thèse de doctorat, université Bordeaux-I, Bordeaux, $347 \mathrm{p}$

11. Schmitt A (2002) Estimation de l'âge au décès des sujets adultes à partir du squelette : des raisons d'espérer. Bull Mem Soc Anthropol Paris 14:51-73

12. Bruzek J (1991) Fiabilité des procédés de détermination du sexe à partir de l'os coxal. Implication à l'étude du dimorphisme sexuel de l'Homme fossile. Thèse de doctorat, Institut de paléontologie humaine et Muséum d'histoire naturelle, Paris, 390 p

13. Sefcáková A, Mizera I, Thurzo M (1999) New human fossil remains from Slovakia. The skull from Moca (late upper Paleolithic, South Slovakia). Bulletin Slovenskej Antropologickej Spolecnosti 2:55-63

14. Sládek V, Sefcáková A, Bruzek J (2001) Sex dimorphism among the early upper paleolithic hominids from central Europe: cranial and pelvic metric variation. J Hum Evol 40:A23

15. Bruzek J, Sefcakova A, Cerny V (2004) Révision du sexe des squelettes épipaléolithiques de Taforalt et d'Afalou-bou-Rhoummel par une approche probabiliste. Antropo 7:195-202
16. Gambier D, Bruzek J, Schmitt A, et al (2006) Révision du sexe et de l'âge au décès des fossiles de Cro-Magnon (Dordogne, France) à partir de l'os coxal. C R Palevol 5:735-41

17. Bruzek J, Schmitt A, Murail P (2005) Identification biologique individuelle en paléoanthropologie. Détermination du sexe et estimation de l'âge au décès à partir du squelette. In: Dutour $\mathrm{O}$, Hublin JJ, Vandermeersch B (eds) Objets et méthodes en paléoanthropologie. Éditions du CTHS, Paris, pp 217-46

18. Webb PA, Suchey JM (1985) Epiphyseal union of the anterior iliac crest and medial clavicle in a modern multiracial sample of American males and females. Am J Phys Anthropol 68:457-66

19. Schmitt A (2005) Une nouvelle méthode pour estimer l'âge au décès des adultes à partir de la surface sacropelvienne iliaque. Bull Mem Soc Anthropol Paris 17:89-101

20. Henry-Gambier D, Bruzek J, Schmitt A, et al (2007) Changement de paradigme dans la détermination du sexe et de l'âge au décès des sujets adultes à partir du squelette : application aux fossiles du Paléolithique supérieur d'Europe. In: Evin J (ed) Un siècle de construction du discours scientifique en Préhistoire, vol. 3. Actes du $26^{\mathrm{e}}$ Congrès de la SPF, Avignon, 2004. SPF, Paris, pp 515-25

21. Villotte S (2009) Enthésopathies et activités des hommes préhistoriques. Recherche méthodologique et application aux fossiles européens du Paléolithique supérieur et du Mésolithique. Archaeopress, Oxford, $206 \mathrm{p}$

22. Smith BH (1984) Patterns of molar wear in hunter-gatherers and agriculturalists. Am J Phys Anthropol 63:39-56

23. Bruzek J (2002) A method for visual determination of sex, using the human hip bone. Am J Phys Anthropol 117: 157-68

24. Murail P, Bruzek J, Houët J, et al (2005) DSP: a tool for probabilistic sex diagnosis using worldwide variability in hip-bone measurements. Bull Mem Soc Anthropol Paris 17:167-76

25. Henry-Gambier D, Bruzek J, Murail P, et al (2002) Révision du sexe du squelette magdalénien de Saint-Germain-la-Rivière (Gironde, France). Paleo 14:205-12

26. Jones ER, Gonzalez-Fortes G, Connell S, et al (2015) Upper Palaeolithic genomes reveal deep roots of modern Eurasians. Nat Com 6:8212

27. Fu Q, Posth C, Hajdinjak M, et al (2016) The genetic history of Ice Age Europe. Nature 534:200-5

28. Tarsi T, Noto F, Martinez-Labarga C, et al (2006) Ricostruzione della storia genetica per via materna delle comunità paleolitiche delle grotte dei Balzi Rossi, della Caverna delle Arene Candide e di Grotta del Romito e di quelle neolitiche ed eneolitiche di Samari e di Fontenoce di Recanati. In: Martini AF (ed) La cultura del morire nelle società preistoriche e protostoriche italiane, Origines. Istituto Italiano di Preistoria e Protostoria, Firenze, pp 315-346

29. Martin R (1928) Lehrbuch der Anthropologie in systematischer Darstellung, vol. 2: Kraniologie. Osteologie. Gustav Fischer, Jena, $604 \mathrm{p}$

30. Murail P, Bruzek J, Braga J (1999) A new approach to sexual diagnosis in past populations. Practical adjustments from Van Vark's procedure. Int J Osteoarchaeol 9:39-53 